\title{
Infectious complications in children with malignant bone tumors: a multicenter nationwide study
}

This article was published in the following Dove Press journal:

Infection and Drug Resistance

Krzysztof Czyzewski iD', Przemyslaw Galazka ${ }^{2}$, Patrycja

Zalas-Wiecek ${ }^{3}$, Olga GryniewiczKwiatkowska ${ }^{4}$, Agnieszka Gietka ${ }^{4}$, Katarzyna Semczuk ${ }^{5}$, Liliana

Chelmecka-Wiktorczyk ${ }^{6}$, Iwona

$\mathrm{Zak}^{7}$, Malgorzata Salamonowicz, Jowita Fraczkiewicz ${ }^{8}$, Olga ZajacSpychala ${ }^{9}$, Ewa Bien ${ }^{10}$, Marcin Plonowski '1, Pawel Wawrykow ${ }^{12}$, Filip Pierlejewski ${ }^{13}$, Zuzanna

Gamrot $^{14}$, Zofia Malas ${ }^{15}$,

Weronika Stolpa ${ }^{16}$, Jakub Musial ${ }^{17}$, Jan Styczynski

'Department of Pediatric Hematology and Oncology, Collegium Medicum, Nicolaus Copernicus University Torun, Bydgoszcz, Poland; ${ }^{2}$ Department of General and Oncological Surgery for Children and Adolescents,

Collegium Medicum, Nicolaus Copernicus University

Torun, Bydgoszcz, Poland; ' 3 Department of

Microbiology, Collegium Medicum, Nicolaus

Copernicus University Torun, Bydgoszcz, Poland;

${ }^{4}$ Department of Oncology, Children's Memorial Health

Institute, Warsaw, Poland; ${ }^{5}$ Department of

Microbiology, Children's Memorial Health Institute,

Warsaw, Poland; ' ${ }^{6}$ Department of Pediatric Oncology

and Hematology, University Children's Hospital,

Jagiellonian University, Collegium Medicum, Krakow.

Poland; ${ }^{7}$ Department of Microbiology, University

Children's Hospital, Jagiellonian University, Collegium

Medicum, Krakow, Poland; ${ }^{8}$ Department of Pediatric

Stem Cell Transplantation, Hematology and Oncology,

Medical University, Wroclaw, Poland; 'Department of

Pediatric Oncology, Hematology and Transplantology,

University of Medical Sciences, Poznan, Poland

${ }^{10}$ Department of Pediatrics, Hematology and

Oncology, Medical University, Gdansk, Poland;

"Department of Pediatric Oncology and Hematology, Medical University, Bialystok, Poland; ${ }^{12}$ Department of Pediatrics and Pediatric Oncology, Pomeranian Medical University, Szczecin, Poland; ${ }^{13}$ Department of Pediatric Oncology and Hematology, Medical University, Lodz, Poland; ${ }^{4}$ Division of Pediatric Hematology and Oncology, Chorzow City Hospital, Chorzow, Poland; ${ }^{15}$ Division of Pediatric Hematology and Oncology, Children Hospital, Olsztyn, Poland; ${ }^{16}$ Division of

Pediatric Oncology, Hematology and Chemotherapy, Department of Pediatric, Silesian Medical University, Katowice, Poland; ${ }^{17}$ Department of Pediatric

Oncohematology, Medical Faculty University of Rzeszow, Clinical Provincial Hospital No. 2, Rzeszow, Poland

Correspondence: Krzysztof Czyzewski Department of Pediatric Hematology and Oncology, Collegium Medicum, Nicolaus

Copernicus University Torun, ul. Sklodowskiej-

Curie 9, Bydgoszcz 85-094, Poland

Tel +48525854860

Fax +48525854087

Email k.czyzewski@cm.umk.pl
Objectives: The analysis of epidemiology, risk factors and outcome of infections in children with malignant bone tumors (MBT) undergoing chemotherapy.

Methods: In this retrospective nationwide multicenter cross-sectional study, a total number of 126 children with MBT including 70 with Ewing sarcoma (ES) and 56 with osteosarcoma (OSA) were screened for infections over a period of 72 consecutive months.

Results: The risk of infection was 7.15-fold higher in patients with ES as compared to the OSA group, especially concerning bacterial infections (4.1-fold increase risk). Bacterial infections occurred in $74.3 \%$ patients with ES and in $41.1 \%$ with OSA. The median time from diagnosis to first infection was 4.9 months. 33.0\% of bacterial episodes were diagnosed as bloodstream (BSI), $31.1 \%$ as gastrointestinal tract, $30.1 \%$ as urinary tract infection. Infection-related mortality (IRM) from bacterial infection was $6 \%$ and $15 \%$ in ES and OSA patients, respectively. Cumulative incidence was $7.1 \%$ for invasive fungal disease and $6.3 \%$ for viral infections. The only significant risk factor for IRM was time to infection $\geq 5$ months since the beginning of chemotherapy. All patients who have died from infection had BSI and were in neutropenia.

Conclusions: Infections in the children with MBT in our study occurred with high frequency, especially in patients with ES. The most frequent were bacterial infections, while fungal and viral infections were episodic. Among the bacterial infections, bloodstream, urinary tract and gastrointestinal tract infections occurred with similar frequency. All deceased patients died due to BSI. Bacterial infection occurring $\geq 5$ months since the beginning of chemotherapy was a risk factor for death.

Keywords: infectious complications, risk factors analysis, Ewing sarcoma, osteosarcoma, malignant bone tumor, children

\section{Introduction}

Infections are the major cause of morbidity and mortality in patients with cancer or undergoing hematopoietic cell transplantation (HCT). ${ }^{1-3}$ Children with malignancies are highly susceptible to bacterial, fungal and viral infections as a consequence of intensive chemotherapy, impairment of the immune system, alterations in the body's natural barriers, or presence of central venous catheters. ${ }^{1}$ Malignant bone tumors $(\mathrm{MBT})$ in children are a rare group of tumors that accounts for $3-5 \%$ of pediatric cancer below 18 years of age. ${ }^{4}$ The two most frequent MBT are osteosarcoma (OSA) (51\%) and Ewing sarcoma (ES) $(45.9 \%){ }^{4}$ The current standard of management for ES is a combination treatment based on: neo-adjuvant chemotherapy, surgery, adjuvant chemotherapy with high-dose chemotherapy followed by autologous HCT (auto-HCT) in the case of IV stage, and radiotherapy. ${ }^{5,6}$ In patients 
with ES, the duration of combined oncological treatment ranges from 8-9 months for patients treated with highdose chemotherapy followed by auto-HCT to 14 months in patients treated without auto-HCT. Treatment of OSA includes neo-adjuvant chemotherapy, surgery and adjuvant chemotherapy. ${ }^{5}$ Radiotherapy does not apply in OSA, and surgery might be recommended in cases of metastases. ${ }^{5,7}$ In patients with OSA, the duration of the combined oncological treatment ranges from 9 months for good-responde rs to 11-12 months for poor responders.

There are limited data on bacterial, fungal and viral infections in children with ES and OSA during intensive anticancer treatment. The objective of this study was to assess the epidemiology, risk factors and outcome of infections in children with MBT.

\section{Methods}

The study was designed as a retrospective multicenter nationwide cross-sectional analysis performed on behalf of the Polish Society of Pediatric Oncology and Hematology. Bacterial, fungal and viral infections diagnosed in the 6-year period (between January 1, 2012 and December 31, 2017), were reported by each of 13 participating centers and data were analyzed centrally by two independent researchers. In patients who underwent high-dose chemotherapy with HCT the observation was completed on the day before conditioning regimen to transplantation was started.

Only microbiologically documented bacterial infections (MDI) were analyzed. The following clinical categories of infections were diagnosed: bloodstream infections (BSI - presence of viable bacteria in bloodstream, combined with relevant clinical symptoms), urinary tract infections (UTI - presence of viable bacteria in urinary tract, combined with relevant clinical symptoms), skin and soft tissue infections (SSTI - primary or secondary cutaneous or soft tissue involvement due to any infectious bacterial agent) and gut infection (GI a combination of clinical symptoms from the gastrointestinal tract, and demonstration of bacteria and viruses in a specimen from the gastrointestinal tract by culture, antigen detection or PCR). ${ }^{1,8}$ Colonization was not included into this analysis. Bacteria were analyzed also with respect to their resistance profile: ESBL (extended-spectrum $\beta$-lactamase), AmpC (cephalosporinase AmpC), KPC (Klebsiella pneumoniae carbapenemase; class A carbapenemase), MBL (metallobeta-lactamase; class B carbapenemase), OXA-48 (oxacillinase-48; class D carbapenemase) in Gram-negative rods, MRS (methicillin-resistant Staphylococcus), VRE (vancomycin-resistant Enterococcus), LRE (linezolidresistant Enterococcus) and HLAR (high level aminoglycoside-resistant Enterococcus). ${ }^{1,9}$ Carbapenemases were detected by phenotypic and genotypic methods of identification, while other mechanisms of resistance by phenotypic methods. Multidrug-resistant (MDR) bacteria were defined as acquired non-denote resistance to at least two antibiotics used in empiric therapy or resistance to at least three antibiotic classes. ${ }^{1,10,11}$

The invasive fungal disease (IFD) was diagnosed according to European Organizations for Research and Treatment of Cancer/Mycoses study group (EORTC/MSG) criteria as proven, probable and possible. ${ }^{12,13}$ Infections of urinary tract with Candida species were diagnosed as probable. Viral infections were classified as latent or episodic. ${ }^{14}$ Respiratory tract infections were diagnosed with PCR, while GI infections with antigen-based tests. No routine pharmacological anti-infective prophylaxis was used in children with MBT during oncological treatment. Commonly accepted strategies were performed for empirical and targeted anti-infectious therapy with various agents. $1,11,15,16$ Neutropenia was defined as absolute neutrophil count lower than $1500 / \mu \mathrm{L}$, and severe neutropenia $<500 / \mu \mathrm{L}$.

\section{Statistical analysis}

The chi-square test was used for categorical variables, and the Mann-Whitney $U$ test for non-categorical variables. Odds ratio (OR) and confidence intervals (CI) were calculated for the differences in frequency of infections in patients. Cumulative incidences of viral, bacterial or fungal infections were calculated using competing risk analysis, ${ }^{17}$ starting from the day of diagnosis of MBT, to the day of the first infection. An event was defined as the diagnosis of a first specific infectious disorder. Death was considered as the competing event. The Kaplan-Meier method was used to determine infection-related mortality (IRM) and overall survival (OS). ${ }^{18}$ The risk factors were analyzed by multivariate logistic regression model in order to estimate hazard ratio (HR) with 95\%CI. All reported $p$-values are two-sided; $p<0.05$ was considered as statistically significant.

\section{Results}

\section{Frequency of infection}

Infectious complications occurred in 83 of $126(65.8 \%)$ children with MBT including 59/70 (84.3\%) with ES and $24 / 56(42.8 \%)$ with OSA $(\mathrm{OR}=7.15,95 \% \mathrm{CI}=3.11-16.46$, 
$p<0.001$ ); 43 boys and 40 girls, with a median age of 13.3 years (range: 16 days to 21 years). The median time from the diagnosis to the first infection (bacterial, fungal or viral) was 4.9 months (range $0.2-20$ ). With respect to etiology, bacterial infections were diagnosed in $85.8 \%$ cases, fungal in $7.6 \%$ and viral in $6.7 \%$ of patients.

\section{Bacterial infections}

A total number of 103 episodes of bacterial infections occurred in 75 of 126 (59.5\%) patients. They included 76/ 103 (73.8\%) episodes in 52/70 (74.3\%) patients with ES and 27/103 (26.2\%) episodes in 23/56 (41.1\%) patients with OSA $(\mathrm{OR}=4.1,95 \% \mathrm{CI}=1.9-8.8, p<0.001)$. The cumulative incidence of microbiologically-documented bacterial infections is shown in Figure 1. The median time from the diagnosis to the first episode of bacterial infection was 5.4 months (range $0.1-20$ months). According to the site of infection, $33.0 \%$ of bacterial episodes were diagnosed as BSI, $31.1 \%$ as GI, $30.1 \%$ as UTI, and $6 \%$ as SSTI. All SSTI were associated with post-operative wound infection after surgical procedure. Site of infection and type of pathogen was shown in Table S1. Overall, $27.2 \%$ of the patients had more than one bacterial MDI, including two infections in $19.4 \%$ of the patients and three infections in $7.8 \%$ of the patients. None of the patients had more than three bacterial MDI. In 7.8\% of episodes of infection more than one strain was isolated from the same specimen (seven episodes with two strains (UTI; 4, BSI; 2, SSTI; 1) and one episode (SSTI) with four strains). In $4.8 \%$ $(5 / 103)$ episodes coexistence of infection in two sites was diagnosed (UTI/BSI - three episodes, UTI/SSTI - one episode, UTI/GI - one episode) (Table S2).

The most frequently diagnosed Gram-negative bacteria were Escherichia coli (40.8\%), Klebsiella pneumoniae (25.3\%) and Pseudomonas aeruginosa (11.3\%). The most frequently diagnosed Gram-positive bacteria were Clostridium difficile (29.8\%), S. epidermidis (21.3\%) and S. aureus (14.9\%) (Tables 1 and 2).

MDR pattern was present in $42.4 \%$ strains $(56.3 \%$ Gram-negative and 21.3\% Gram-positive) and was present in $100.0 \%$ of Enterobacter cloacae, $76.9 \%$ of K. pneumoniae, $66.7 \%$ of Acinetobacter baumannii and $61.9 \%$ of E. coli. The MRS occurred in $17.6 \%$ of all staphylococcal infections and vancomycin resistance was identified in $25.0 \%$ of enterococcal infections (Tables 1 and 2). MDR Gram-negative bacteria were more frequent in $\mathrm{ES}$ than in OSA $(\mathrm{OR}=3.4,95 \% \mathrm{CI} 1.15-10.1, p=0.027)$

The median time of treatment of bacterial infections was 10 days (range $0-46$ days) and was the same for ES and OSA patients. In patients with ES, IRM was $0.06(95 \%$ $\mathrm{CI}=0.02-0.1)$ and did not differ from patients with OSA $(0.15,95 \% \mathrm{CI}=0.07-0.23)$ (Figure 2). All deceased patients (six cases) died due to BSI (with coexistence of UTI in

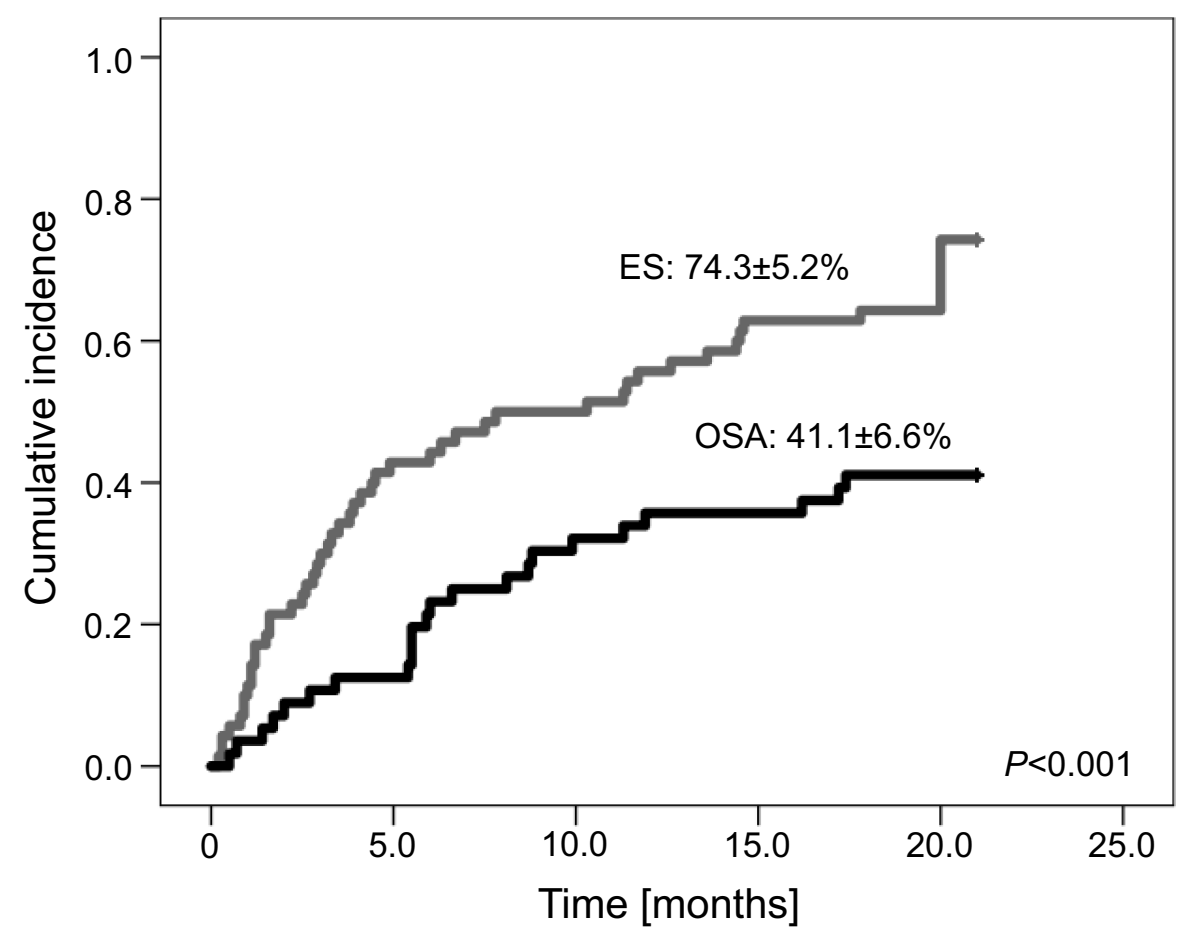

Figure I Bacterial infection incidence in patients with Ewing Sarcoma (ES) and osteosarcoma (OSA). 

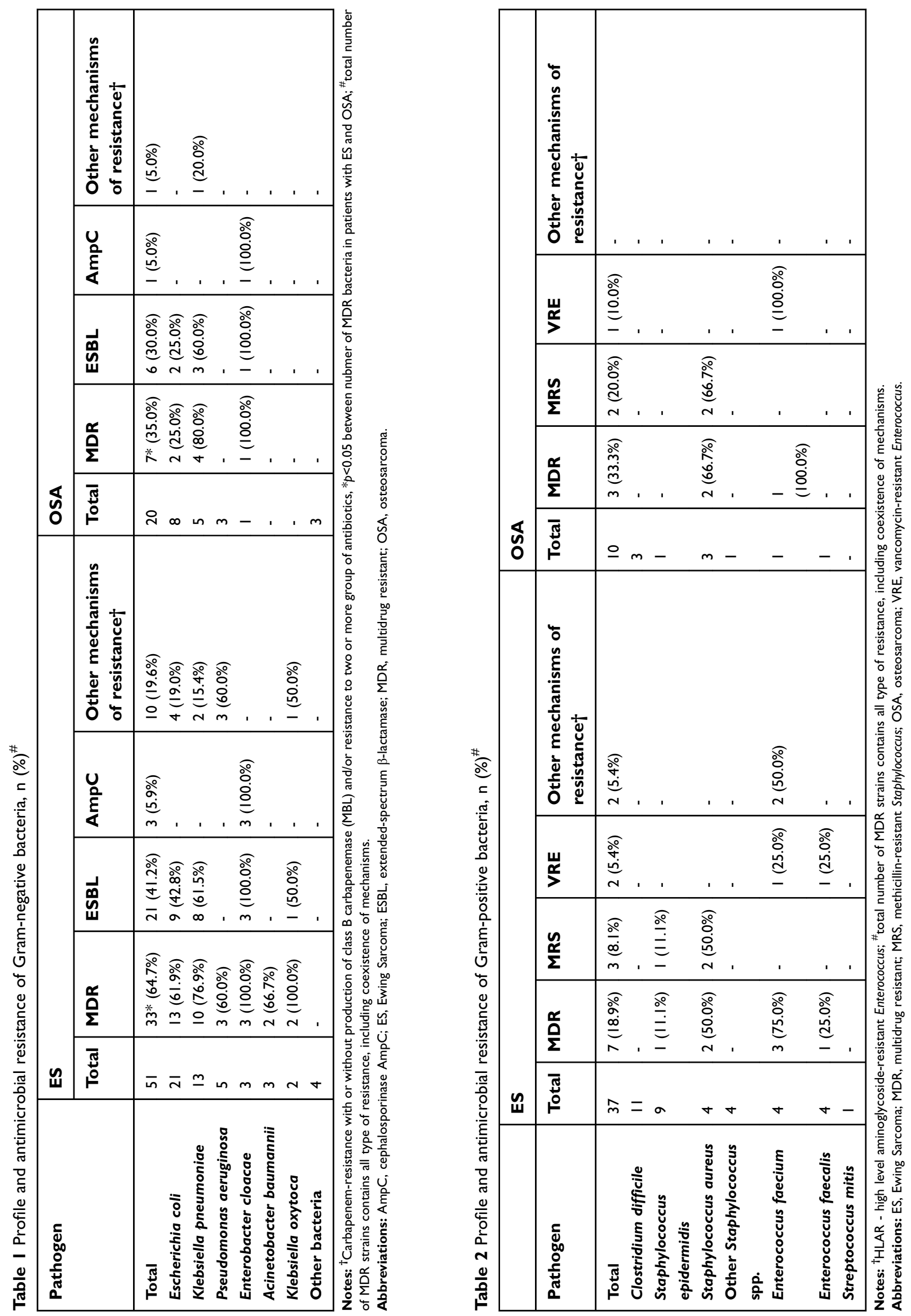


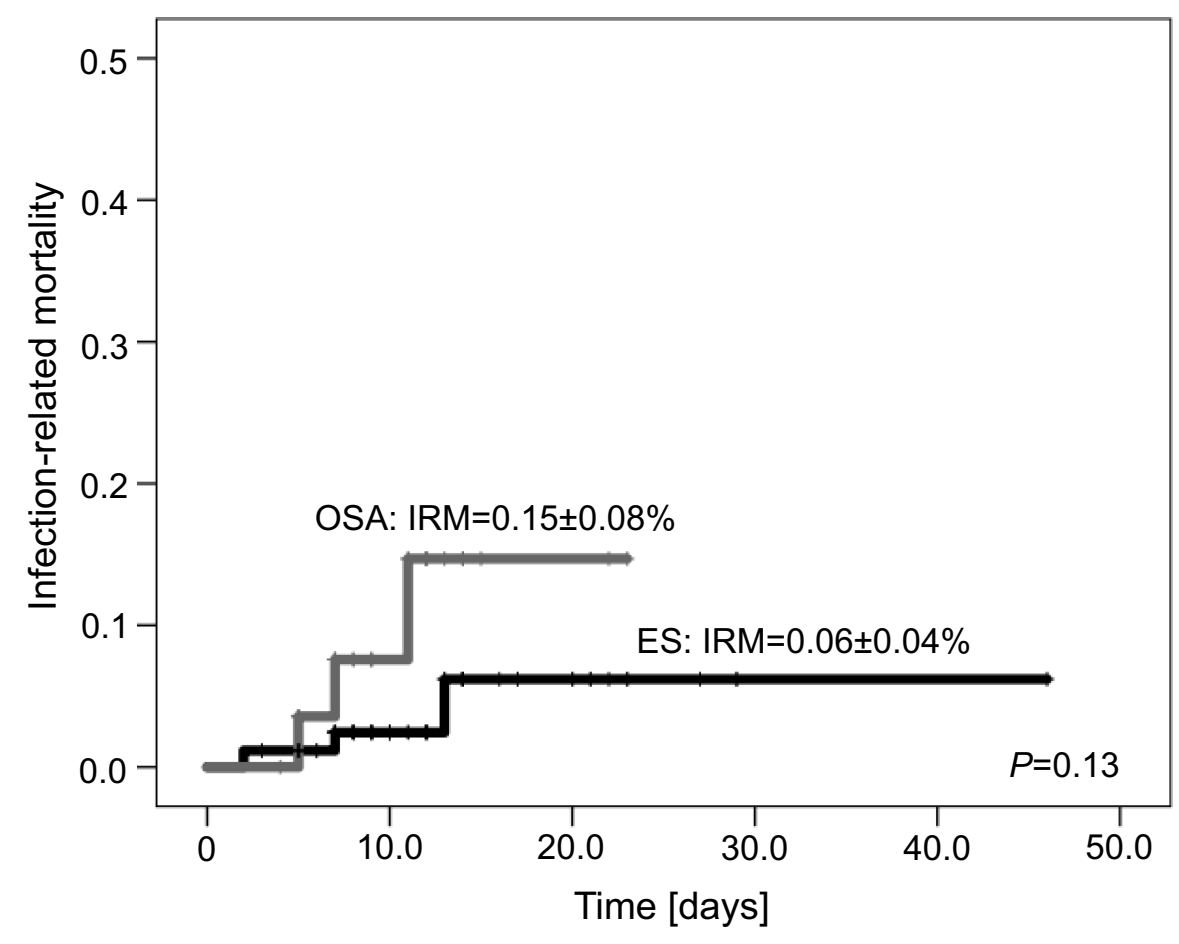

Figure 2 Infection related mortality (IRM) in patients with Ewing Sarcoma (ES) and osteosarcoma (OSA).

33.3\%) during neutropenia. In two of these patients MDR bacteria were cultured. During the study period we observed six deaths $(6 / 126=4.8 \%)$ not related to infection, due to disease progression $(5 / 70=7.1 \%$ with ES and $1 / 56=1.8 \%$ with OSA).

\section{Fungal infections}

Cumulative incidence of IFD was $7.1 \%$ (nine patients), including proven IFD in $0.8 \%$ (one patient), probable $2.4 \%$ (three patients) and possible in 3.9\% (five patients). None of the patients was diagnosed more than once for IFD; $22.2 \%$ of the IFD were reported in OSA (all possible), while $77.8 \%$ of the IFD in ES ( $p=$ ns). Clinical manifestation of IFD were: fungemia (one proven case, Candida guilliermondii in ES patient), UTI (two probable cases: one Candida kefyr, one Candida spp., both in ES), pneumonia (six possible IFD: four in ES, two in OSA). The diagnosis of possible fungal pneumonia was based on the chest HRCT in a neutropenic patient with cough and fever lasting $>96$ hours, with no improvement after broadspectrum antibiotic therapy. The median time from the beginning of oncological treatment to IFD diagnosis was 6.2 months (range 0.6-20 months). The median time of anti-fungal treatment was 18 days (range 7-104 days): in $66.7 \%$ monotherapy was applied (fluconazole in three cases for 14-71 days, voriconazole in two cases for 33 and 104 days) and caspofungin in one case for 13 days).
Combination therapy was used in the $33.3 \%$ of IFD (one case voriconazole + micafungin for 7 days, one case voriconazole + amphotericin $\mathrm{B}$ for 12 days and one case of caspofungin + amphotericin B for 36 days). All patients survived from IFD.

\section{Viral infections}

Cumulative incidence of viral infection was $6.4 \%$ (eight cases) including $0.8 \%$ of infections with HHV-6 (one case), $4.8 \%$ (six cases) of GI infections (five cases of rotavirus (three ES, two OSA) and one case of norovirus (ES)) and $0.8 \%$ ( 1 case, OSA) of influenza A. The median time from the diagnosis of MBT to diagnosis of viral infection was 2.4 months (range $0.7-13.4$ months). HHV6 infection was treated with ganciclovir for 8 days, and influenza A infection with oseltamivir for 5 days. In all cases symptomatic treatment was also implemented. No patient died due to the viral infections.

\section{Risk factor analysis}

The following risk factors predisposing to the incidence of infection were analyzed: primary diagnosis, sex, age $(<13$ years vs $\geq 13$ years), and neutropenia. No risk factor was significant for bacterial, fungal and viral infections.

As no patient died from fungal or viral infection, the risk factor analysis for IRM was performed only for bacterial 
infections. The following risk factors were analyzed for bacteria-related IRM: primary diagnosis, sex, age $(<13$ years vs $\geq 13$ years), type of bacteria (Gram-negative vs Grampositive), neutropenia, bacteremia, pneumonia, time to infection ( $<5$ months vs $\geq 5$ months), and duration of treatment of infection $(<11$ days $v s \geq 11$ days). The only significant risk factor for death was time to infection $\geq 5$ months since primary diagnosis of bone tumor (Table 3, Figure 3). A trend towards higher mortality in patients $\geq 13$ years was observed. All patients who died from bacterial infection had BSI and were neutropenic for a median of 7 days (range 2-14).

Table 3 Multivariate logistic regression analysis for risk factors for death from bacterial infections

\begin{tabular}{|l|l|l|}
\hline Risk factor & HR (95\%CI) & P \\
\hline Primary diagnosis: OSA vs ES & $2.5(0.5-14)$ & 0.293 \\
Sex: male vs female & $1.1(0.8-1.3)$ & 0.961 \\
Severe neutropenia & $3.2(0.8-16)$ & 0.108 \\
Age: $\geq 13$ years vs $<13$ years & $6.9(0.7-62)$ & 0.092 \\
Bacteria: Gram-negative vs Gram-positive & $1.6(0.7-2.4)$ & 0.430 \\
Time to infection since diagnosis: $\geq 5$ vs & $9.7(1.1-96)$ & $\mathbf{0 . 0 4 7}$ \\
<5 months & & \\
Treatment duration of infection: $\geq 1$ I vs & $0.3(0.1-2.0)$ & 0.224 \\
$<1$ । days & & \\
\hline
\end{tabular}

Notes: $* p<0.05$ between time to infection since diagnosis: $\geq 5$ months and $<5$ months are in bold.

Abbreviations: $\mathrm{HR}$, hazard ratio; $\mathrm{Cl}$, confidence intervals; OSA, osteosarcoma; ES, Ewing sarcoma.

\section{Discussion}

Introduction of chemotherapy in order to intensify treatment together with the use of radiotherapy and surgical therapy of MBT has resulted in a significant increase in curability. ${ }^{5}$ The main adverse effects of intensification chemotherapy are neutropenia and infectious complications. ${ }^{1}$ There are only a few reports on infectious complications in children with MBT (apart from case reports) which focus on infectious complications in the perioperative period, prosthetic infections or surgical site infections. ${ }^{19-22}$

We report the results of a study on epidemiology, risk factors and outcome of infections in children with ES or OSA including bacterial, fungal and viral episodes analyzed over a period of 6 years. All reported patients were treated with the same therapeutic protocols, using comparable supportive therapies.

The highest risk of infectious complications related with the applied chemotherapy occurs in patients with acute leukemia, non-Hodgkin's lymphoma and after HCT. ${ }^{1,5}$ We observed high incidence of infectious complications in children with MBT, especially with ES. More advanced stages at diagnosis, more intensive chemotherapy with more immunosuppressive reagents and more MDR bacteria were the possible reason of higher incidence of infectious complications in ES than OSA. Analysis of neutropenic fever after the VIDE cycles in

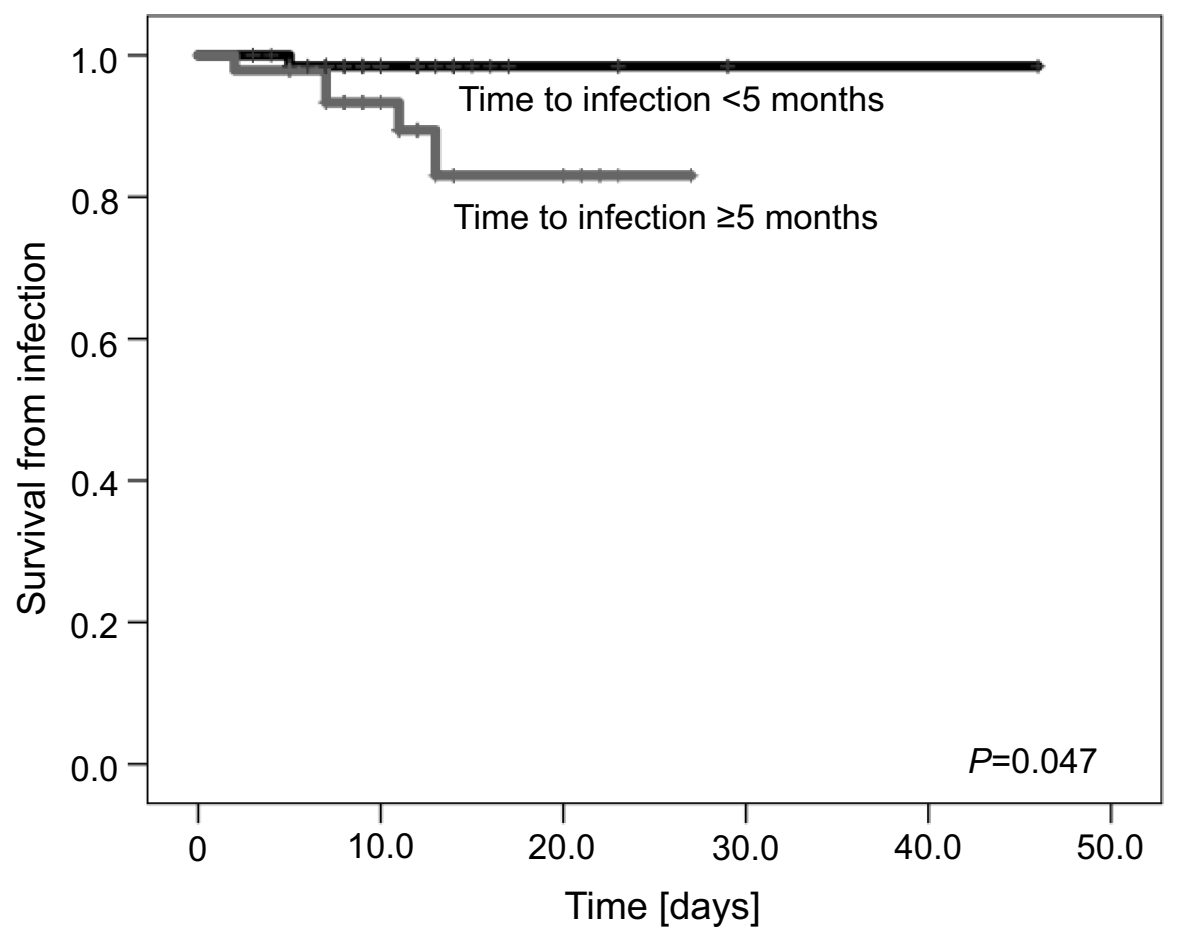

Figure 3 Survival from bacterial infection with respect to time of infection $(<5$ months vs $\geq 5$ months from the primary diagnosis of bone tumor). 
patients treated according to EURO-EWING 99 was performed by Penel-Page et al. ${ }^{23}$ It was shown that patients treated in the adult services were less frequently hospitalized with shorter length of stay, without differences in survival, number of documented infections, transfusions, dose modification, delay in chemotherapy, or stay in the intensive care unit. $^{23}$

Angelini et $\mathrm{al}^{24}$ analyzed infectious complications in patients after surgical resection due to bone tumors. They found that $20 \%$ of patients (15\% without bone reconstruction and $26 \%$ with bone reconstruction) had a deep infection develop at a mean time of 8 months from diagnosis. In the multivariate analysis, only bone surgery was a risk factor for the occurrence bacterial complications. ${ }^{24}$ Longhi et $\mathrm{al}^{25}$ found that among patients with OSA, infection of the central catheter with fever were found in 5.6\% of them. Authors suggest that the most common complications of the use of central venous catheters were infection and venous thrombosis whereas pulmonary septic emboli were rare. ${ }^{25}$ We observed that only $6 \%$ of infections were associated with post-operative wound infection after surgical procedure, while BSI and the UTI were the most frequent type of infection complication.

Additionally, in the analysis of the Polish Pediatric Group for Solid Tumors performed 20 years ago, including infections in patients treated for OSA between 1989 and 1998 according to the protocol OS-SFOP-94, deaths were observed in 5 of 28 treated patients, however only one child died due to infectious complications, while the other four died due to non-infectious complications (three with disease progression/relapse, one because of treatment refusal). ${ }^{26}$

In the other study, among 125 children and young adults with OSA chronic localized infections were determined in $4.8 \%$ of patients. ${ }^{27}$ More amputations $(p<0.001)$ were necessary in infected patients due to uncontrolled infection. The 5-year overall survival rate and event-free survival rate in infected patients were $100 \%$, which were significantly higher than that of the non-infected patients, of whom the rates were $54 \%$ and $43 \%$ respectively $(p=0.01)$. Authors suggest that association between infection and survival rate of OS patients remains to be elucidated, because of the wide spectrum of immune cells and transducers which have shown potential in the treatment of OSA. On the other hand, risk factors analysis showed that only tumor metastasis was an independent negative risk factor for survival $(p<0.001) .{ }^{27}$ In our study in the multivariate analysis for risk factors of death from bacterial infection we found that the only significant risk factor was time to infection $\geq 5$ months since primary diagnosis of MBT and severe neutropenia was present in all these cases for a period of 2-14 days. However, neutropenia was not a significant factor in the multivariate analysis, as most bacterial infections developed during the neutropenic phase. High intensity of the chemotherapy followed by myelosuppression could be the reasons for this observation.

We found that the overall cumulative incidence of bacterial infections in ES and OSA (74.3\% and $41.1 \%$, respectively) was higher than previously reported in patients with acute lymphoblastic leukemia (ALL) (40.9\%), Non-Hodgkin lymphoma (NHL) (37.0\%) and Hodgkin's disease (HD) (12.7\%), while in acute myelogenous leukemia (AML) it was comparable $(58.0 \%) .{ }^{1} \mathrm{We}$ have shown that IFD incidence in patients with MBT was lower $(7.1 \%)$ than in patients after allo-HCT $(30.6 \%)$ and auto-HCT (17.1\%) and also in patients with AML (43.0\%), ALL (13.0\%) and NHL (10.2\%), but higher than in patients with HD $(1.8 \%){ }^{1}$ Comparing to HCT patients, ${ }^{1}$ MDR bacteria were less frequent in patients with MBT ( $76.8 \%$ vs $42.4 \%$ respectively), what can be explained by usually longer and more intensive chemotherapy preceding HCT.

Although the present study is inherent to several limitations of observational research, including retrospective design, its strong points are that it is a multicenter, nationwide long-term study carried out on a large homogeneous group of patients treated in the same way in all centers. Additionally, to our knowledge, this is the first such comprehensive study of infectious complications in children with MBT.

\section{Conclusions}

Infections in children with MBT occurred with high frequency, especially in patients with ES. The most frequent were bacterial infections, while fungal and viral infections were episodic. Among the bacterial infections, BSI, UTI and GI occurred with similar frequency. The MDR bacteria occurred in $42.4 \%$ of episodes, and mainly they were Gram-negative bacteria and were more frequent in ES patients than OSA patients. All patients who died from infection were in severe neutropenia and died due to BSI. The only significant risk factor for death was time to infection $\geq 5$ months since primary diagnosis of MBT in patients with neutropenia. Presented data provide new insights into infections in children with MBT and 
underline that there is an increased risk of death related to infection after 5 months of intensive chemotherapy.

\section{Abbreviation list}

ALL, acute lymphoblastic leukemia; AML, acute myelogenous leukemia; AmpC, cephalosporinase AmpC; auto-HCT, autologous hematopoietic cell transplantation; BSI, bloodstream infections; CI, confidence intervals; EORTC/MSG, European Organizations for Research and Treatment of Cancer/Mycoses study group; ES, Ewing sarcoma; ESBL, extended-spectrum $\beta$-lactamase; GI, gut infection; HCT, hematopoietic cell transplantation; HD, Hodgkin's disease; HHV-6, human herpesvirus 6; HLAR, high level aminoglycoside-resistant Enterococcus; IFD, invasive fungal disease; IRM, infection-related mortality; KPC, Klebsiella pneumoniae carbapenemase, class A carbapenemase; LRE, linezolidresistant Enterococcus; MBL, metallo-beta-lactamase, class B carbapenemase; MBT, malignant bone tumors; MDI, microbiologically documented bacterial infections; MDR, multidrug resistant; MRS, methicillin-resistant Staphylococcus; NHL, Non-Hodgkin lymphoma; OR, odds ratio; OS, overall survival; OSA, osteosarcoma; OXA-48, oxacillinase-48, class D carbapenemase; SSTI, skin and soft tissue infections; UTI, urinary tract infections; VRE, vancomycin-resistant Enterococcus.

\section{Ethical consideration}

This study was conducted in accordance with the Declaration of Helsinki and approved by the ethics committee of Collegium Medicum in Bydgoszcz, Nicolaus Copernicus University in Torun. All patient data were analyzed in anonymity. As neither individual data were published nor any intervention was performed on patients, patient consent was waived by the ethics committee.

\section{Acknowledgments}

The authors thank the following persons for their support and contribution to the creation of this paper: Magdalena Dziedzic, Monika Pogorzala, Ewa Demidowicz, Natalia Bartoszewicz, Mariusz Wysocki, Bozenna Dembowska-Baginska, Katarzyna Dzierzanowska-Fangrat, Walentyna Balwierz, Bernarda Kazanowska, Jacek Wachowiak, Elzbieta Adamkiewicz-Drozynska, Maryna Krawczuk-Rybak, Jaroslaw Peregud-Pogorzelski, Wojciech Mlynarski, Mariola Woszczyk, Wanda Badowska, Grazyna Sobol-Milejska, Radoslaw Chaber. The authors thank anonymous reviewers for their suggestions to improve the article.

\section{Author contributions}

$\mathrm{KC}$ and JS were responsible for the concept of the study and design. $\mathrm{KC}$ was the major contributor in writing the paper. All authors contributed toward data collection, data analysis, critically revising the paper, gave final approval of the version to be published and agree to be accountable for all aspects of the work.

\section{Disclosure}

The authors report no conflicts of interest in this work.

\section{References}

1. Styczynski J, Czyzewski K, Wysocki M, et al. Increased risk of infections and infection-related mortality in children undergoing haematopoietic stem cell transplantation compared to conventional anticancer therapy: a multicentre nationwide study. Clin Microbiol Infect. 2016;22(2):179 e171-179 e110. doi:10.1016/j. cmi.2015.10.017

2. Gooley TA, Chien JW, Pergam SA, et al. Reduced mortality after allogeneic hematopoietic-cell transplantation. $N$ Engl $J$ Med. 2010;363(22):2091-2101. doi:10.1056/NEJMoa1004383

3. Choi YB, Yi ES, Kang JM, et al. Infectious complications during tandem high-dose chemotherapy and autologous stem cell transplantation for children with high-risk or recurrent solid tumors. PLoS One. 2016;11(9):e0162178. doi:10.1371/journal.pone.0162178

4. Kaatsch P, Strothotte J, Becker C, Bielack S, Dirksen U, Blettner M. Pediatric bone tumors in Germany from 1987 to 2011: incidence rates, time trends and survival. Acta Oncol. 2016;55(9-10):1145-1151. doi:10.1080/0284186X.2016.1195509

5. Pogorzala M, Styczynski J, Czyzewski K, et al. [Infectious complications in children with bone tumors]. Postepy N Med. 2016;29 (8):551-554.

6. Ladenstein R, Potschger U, Le Deley MC, et al. Primary disseminated multifocal Ewing sarcoma: results of the Euro-EWING 99 trial. J Clin Oncol. 2010;28(20):3284-3291. doi:10.1200/JCO.2009.22.9864

7. Karwacki MW, Wozniak W, Lugowska I, Rychlowska-Pruszynska M. [The results of a single center pilot study of combined high-dose methotrexate and doxorubicin with cisplatin in neo-adjuvant chemotherapy for osteosarcoma in children and adolescents]. Nowotwory J Oncol. 2010;60(5):425-432.

8. European Commission. Commission Implementing Decision of 8 August 2012 amending Decision 2002/253/EC laying down case definitions for reporting communicable diseases to the Community network under Decision No 2119/98/EC of the European Parliament and of the Council (2012/506/EU). Official Journal of the European Union. Luxembourg: Publications Office of the European Union. 2012;L262:1-57.

9. Bassetti M, Righi E. Multidrug-resistant bacteria: what is the threat? Hematology Am Soc Hematol Educ Program. 2013;2013:428-432. doi:10.1182/asheducation-2013.1.428

10. Averbuch D, Cordonnier C, Livermore DM, et al. Targeted therapy against multi-resistant bacteria in leukemic and hematopoietic stem cell transplant recipients: guidelines of the 4th European Conference on Infections in Leukemia (ECIL-4, 2011). Haematologica. 2013;98 (12):1836-1847. doi:10.3324/haematol.2013.091330

11. Averbuch D, Orasch C, Cordonnier C, et al. European guidelines for empirical antibacterial therapy for febrile neutropenic patients in the era of growing resistance: summary of the 2011 4th European Conference on Infections in Leukemia. Haematologica. 2013;98 (12):1826-1835. doi:10.3324/haematol.2013.091025 
12. De Pauw B, Walsh TJ, Donnelly JP, et al. Revised definitions of invasive fungal disease from the European Organization for Research and Treatment of Cancer/Invasive Fungal Infections Cooperative Group and the National Institute of Allergy and Infectious Diseases Mycoses Study Group (EORTC/MSG) Consensus Group. Clin Infect Dis. 2008;46(12):1813-1821. doi:10.1086/588660

13. Groll AH, Castagnola E, Cesaro S, et al. Fourth European Conference on Infections in Leukaemia (ECIL-4): guidelines for diagnosis, prevention, and treatment of invasive fungal diseases in paediatric patients with cancer or allogeneic haemopoietic stem-cell transplantation. Lancet Oncol. 2014;15(8):e327-e340. doi:10.1016/ S1470-2045(14)70017-8

14. Marr KA. Delayed opportunistic infections in hematopoietic stem cell transplantation patients: a surmountable challenge. Hematology Am Soc Hematol Educ Program. 2012;2012:265-270. doi:10.1182/ asheducation-2012.1.265

15. Ljungman $\mathrm{P}$, de la Camara R, Cordonnier C, et al. Management of CMV, HHV-6, HHV-7 and Kaposi-sarcoma herpesvirus (HHV-8) infections in patients with hematological malignancies and after SCT. Bone Marrow Transplant. 2008;42(4):227-240. doi:10.1038/bmt.2008.162

16. Maertens J, Marchetti O, Herbrecht R, et al. European guidelines for antifungal management in leukemia and hematopoietic stem cell transplant recipients: summary of the ECIL 3-2009 update. Bone Marrow Transplant. 2011;46(5):709-718. doi:10.1038/bmt.2010.175

17. Gooley TA, Leisenring W, Crowley J, Storer BE. Estimation of failure probabilities in the presence of competing risks: new representations of old estimators. Stat Med. 1999;18(6):695-706.

18. Kaplan EL, Meier P. Nonparametric estimation from incomplete observations. J Am Stat Assoc. 1958;53(282):457-481. doi:10.1080/ 01621459.1958 .10501452

19. Lee JA, Kim MS, Kim DH, et al. Postoperative infection and survival in osteosarcoma patients. Ann Surg Oncol. 2009;16(1):147-151. doi:10.1245/s10434-008-0184-8
20. Li X, Moretti VM, Ashana AO, Lackman RD. Perioperative infection rate in patients with osteosarcomas treated with resection and prosthetic reconstruction. Clin Orthop Relat Res. 2011;469 (10):2889-2894. doi:10.1007/s11999-011-1877-z

21. Jeys LM, Grimer RJ, Carter SR, Tillman RM, Abudu A. Post operative infection and increased survival in osteosarcoma patients: are they associated? Ann Surg Oncol. 2007;14(10):2887-2895. doi:10.1245/s10434-007-9483-8

22. Campanacci L, Ali N, Casanova JM, Kreshak J, Manfrini M. Resurfaced allograft-prosthetic composite for proximal tibial reconstruction in children: intermediate-term results of an original technique. J Bone Joint Surg Am. 2015;97(3):241-250. doi:10.2106/ JBJS.N.00447

23. Penel-Page M, Normand C, Bertrand A, et al. [Management of febrile neutropenias in adolescents and young adults: differences of practice between adult and pediatric units]. Bull Cancer. 2015;102 (11):915-922. doi:10.1016/j.bulcan.2015.08.001

24. Angelini A, Drago G, Trovarelli G, Calabro T, Ruggieri P. Infection after surgical resection for pelvic bone tumors: an analysis of 270 patients from one institution. Clin Orthop Relat Res. 2014;472 (1):349-359. doi:10.1007/s11999-013-3250-x

25. Longhi A, Rimondi E, Loro L, et al. Pulmonary nodules in osteosarcoma patients: differential diagnosis of central venous catheter-related infections in the lungs. Radiol Med. 2006;111 (2):192-201

26. Chybicka A, Jaworski W, Liebhart M, et al. [The analysis of failures in therapy of osteosarcoma in children treated according to SFOP-94 protocol in the studies of Polish pediatric solid tumors treatment group]. Wiad Lek. 1998;51 Suppl 4:40-44.

27. Chen YU, Xu SF, Xu M, Yu XC. Postoperative infection and survival in osteosarcoma patients: reconsideration of immunotherapy for osteosarcoma. Mol Clin Oncol. 2015;3(3):495-500. doi:10.3892/ mco. 2015.528 


\section{Supplementary materials}

Table SI Site of infection and type of pathogen in patients with ES and OSA

\begin{tabular}{|c|c|c|}
\hline Site & ES & OSA \\
\hline \multicolumn{3}{|l|}{ BSI } \\
\hline $\begin{array}{l}\text { Gram-positive } \\
\text { Gram-negative }\end{array}$ & $\begin{array}{l}\text { Staphylococcus aureus, Staphylococcus epidermidis, Staphylococcus homi- } \\
\text { nis, Staphylococcus lentus, Streptococcus mitis, } \\
\text { Acinetobacter baumannii, Escherichia coli, Enterobacter cloacae, Klebsiella } \\
\text { oxytoca, Klebsiella pneumonia, Pseudomonas aeruginosa, } \\
\text { Stenotrophomonas maltophilia, Panthoea agglomerans }\end{array}$ & $\begin{array}{l}\text { Staphylococcus epidermidis, Staphylococcus lentus } \\
\text { Escherichia coli, Enterobacter cloacae, Klebsiella pneu- } \\
\text { moniae, Leclercia adacarcoxylata, Sphingomonas } \\
\text { paucimobilis }\end{array}$ \\
\hline \multicolumn{3}{|l|}{ GI } \\
\hline $\begin{array}{l}\text { Gram-positive } \\
\text { Gram-negative }\end{array}$ & $\begin{array}{l}\text { Enterococcus faecalis, Clostridium difficile } \\
\text { Acinetobacter baumannii, Escherichia coli, Enterobacter cloacae, Klebsiella } \\
\text { pneumoniae, Pseudomonas aeruginosa }\end{array}$ & $\begin{array}{l}\text { Clostridium difficile } \\
\text { Escherichia coli, Pseudomonas aeruginosa }\end{array}$ \\
\hline \multicolumn{3}{|l|}{ SSTI } \\
\hline $\begin{array}{l}\text { Gram-positive } \\
\text { Gram-negative }\end{array}$ & $\begin{array}{l}\text { Enterococcus faecalis, Staphylococcus aureus, Staphylococcus epidermidis } \\
\text { Acinetobacter baumannii, Escherichia coli, Klebsiella oxytoca, }\end{array}$ & Staphylococcus aureus \\
\hline \multicolumn{3}{|l|}{ UTI } \\
\hline $\begin{array}{l}\text { Gram-positive } \\
\text { Gram-negative }\end{array}$ & $\begin{array}{l}\text { Enterococcus faecium, Enterococcus faecalis, Staphylococcus haemolyticus } \\
\text { Escherichia coli, Klebsiella pneumoniae, Proteus mirabilis, Pseudomonas } \\
\text { aeruginosa }\end{array}$ & $\begin{array}{l}\text { Enterococcus faecalis, Staphylococcus aureus } \\
\text { Escherichia coli, Klebsiella pneumoniae, Proteus mirabilis, } \\
\text { Pseudomonas aeruginosa }\end{array}$ \\
\hline
\end{tabular}

Abbreviations: BSI, bloodstream infections; ES, Ewing sarcoma; GI, gut infections; OSA, osteosarcoma; SSTI, skin and soft tissue infections; UTI, urinary tract infections.

Table S2 Site of infection and type of pathogen in coexistent infections

\begin{tabular}{|l|l|l|}
\hline Episode & Pathogen in first site & Pathogen in second site \\
\hline & UTI & BSI \\
\hline I. & Pseudomonas aeruginosa & Pseudomonas aeruginosa \\
3. & Pseudomonas aeruginosa & $\begin{array}{l}\text { Sseudomonas aeruginosa } \\
\text { Staphylococcus epidermidis }\end{array}$ \\
\hline & Enterococcus faecalis & GI \\
\hline 4. & UTI & Enterococcus faecium \\
\hline & Pseudomonas aeruginosa & SSTI \\
\hline 5. & UTI & Escherichia coli, Klebsiella oxytoca, Enterococcus faecium \\
\hline
\end{tabular}

Abbreviations: BSI, bloodstream infections; GI, gut infections; SSTI, skin and soft tissue infections; UTI, urinary tract infections.

Infection and Drug Resistance

\section{Dovepress}

\section{Publish your work in this journal}

Infection and Drug Resistance is an international, peer-reviewed openaccess journal that focuses on the optimal treatment of infection (bacterial, fungal and viral) and the development and institution of preventive strategies to minimize the development and spread of resistance. The journal is specifically concerned with the epidemiology of antibiotic resistance and the mechanisms of resistance development and diffusion in both hospitals and the community. The manuscript management system is completely online and includes a very quick and fair peerreview system, which is all easy to use. Visit http://www.dovepress.com/ testimonials.php to read real quotes from published authors. 